\title{
LINEAR POTENTIALS AND GALACTIC ROTATION CURVES
}

\author{
Philip D. Mannheim \\ Department of Physics \\ University of Connecticut \\ Storrs, CT 06269-3046 \\ mannheim@uconnvm.bitnet
}

\begin{abstract}
We present a simple, closed form expression for the potential of an axisymmetric disk of stars interacting through gravitational potentials of the form $V(r)=-\beta / r+$ $\gamma r / 2$, the potential associated with fundamental sources in the conformal invariant fourth order theory of gravity which has recently been advanced by Mannheim and Kazanas as a candidate alternative to the standard second order Einstein theory. Using the model we obtain a reasonable fit to some representative galactic rotation curve data without the need for any non-luminous or dark matter. Our study suggests that the observed flatness of rotation curves might only be an intermediate phenomenon rather than an asymptotic one.
\end{abstract}

June, 1993

UCONN-92-3 


\section{(I) Introduction}

In formulating a physical theory it is necessary to both work up from phenomenological observations and down from fundamental principles, and to be prepared to revise the insights obtained from both approaches as new data come on line. However, after a program such as this has been successfully carried through once, there is then some reluctance on the part of the community to have to reopen the issue even in the light of subsequent data. Consequently, the prevailing view on galactic rotation curve data is that their deviation from the behavior expected on the basis of the standard Newton-Einstein theory as applied to the observed galactic luminous matter surface brightness distribution must be attributed to a (rather substantial) non-luminous or dark matter galactic component. Since there is no clear evidence today that the dominant component of the Universe is in fact non-luminous, there is thus some merit in going back over familiar ground to see where, if anywhere, something could be modified. (Milgrom (1983) and Sanders (1990) have also looked at possible revisions to the standard theory, with the recent study of Begeman et al (1991) in particular showing that Milgrom's MOND alternative is currently phenomenologically viable).

Noting that there is currently no known theoretical reason which would select out the standard second order Einstein theory from amongst the infinite class of (all order) covariant, metric based theories of gravity that one could in principle at least consider, Mannheim and Kazanas have reopened the question of what the correct covariant theory of gravity might be (Mannheim and Kazanas (1989, 1991, 1992), Mannheim (1990, 1992, 1993), Kazanas and Mannheim (1991)), and developed an approach which works down from an additional fundamental principle, namely that of local scale or conformal invariance, the invariance now believed to be possessed by the other three fundamental strong, electromagnetic and weak interactions. This invariance forces gravity to be described

uniquely by the fourth order action $I_{W}=-\alpha \int d^{4} x(-g)^{1 / 2} C_{\lambda \mu \nu \kappa} C^{\lambda \mu \nu \kappa}$ where $C_{\lambda \mu \nu \kappa}$ is the conformal Weyl tensor and $\alpha$ is a purely dimensionless coefficient. In their original pa- 
per Mannheim and Kazanas (1989) obtained the exact, non-perturbative exterior vacuum solution associated with a static, spherically symmetric gravitational source such as a star in this theory, viz.

$$
-g_{00}=1 / g_{r r}=1-\beta(2-3 \beta \gamma) / r-3 \beta \gamma+\gamma r-k r^{2}
$$

where $\beta, \gamma$, and $k$ are three appropriate dimensionful integration constants. As they noted, this solution contains the familiar exterior Schwarzschild solution and thereby yields the standard exterior Newtonian potential term and its standard general relativistic corrections whenever the two additional potential terms in Eq. (1) may be ignored. The theory thus contains the same solution as the standard Einstein theory in the appropriate kinematic regime even while not containing the Einstein equations themselves, this being all that observation can require, and thus nicely meets the demands of solar system scale observations. The quadratic term in Eq. (1) may be associated with a general cosmological background de Sitter geometry and is otherwise uneventful, and thus (with both the $3 \beta \gamma$ terms being numerically negligible - see below) the conformal theory leads to the non-relativistic gravitational potential $V(r)=-\beta / r+\gamma r / 2$, which may then be fitted to data whenever the weak gravity limit is applicable. $V(r)$ is thus the potential obtained in coming down from a fundamental principles approach. In this paper we shall study the implications of this potential by working up from galactic data, the first distance scale on which the potential is found to lead to significant deviations from Newton. As we shall see, the two approaches even have a chance to converge; however, those readers who may not be too comfortable with (or even disapprove of) the whole general conformal gravity program can view this paper purely as an attempt to identify which phenomenological potentials the currently available observational data actually permit.

\section{(2) The Potential of an Extended Disk}

For an explicit application of conformal gravity to objects such as galaxies, the treatment completely parallels the Newton-Einstein treatment, i.e. the potential of each of the fundamental stellar constituents of the galaxy is first found by solving the relativis- 
tic theory exactly, with the potential of the galaxy then being found perturbatively by adding up the potentials of these stars in the weak gravity limit. Thus for our purposes here the requisite stellar potential is the above $V(r)=-\beta / r+\gamma r / 2$ with $\gamma$ being a new stellar parameter about which almost nothing is currently known $(\gamma$ is however known to in principle be completely independent of $\beta$ (Mannheim and Kazanas (1992))), with the phenomenological fitting to be presented below providing some first insight into typical numerical values for $\gamma$.

In order to actually determine the weak gravity potential of an extended object such as a disk, we have found it convenient to generalize the approach of Toomre (1963) first to non-thin Newtonian disks (a step also taken by Casertano (1983)), and then to disks with linear potentials. The method which leans heavily on the completeness properties of the $J_{n}(k R)$ Bessel functions will be reported elsewhere, and here we state only the relevant results. The Newtonian potential of a general axisymmetric distribution of stellar matter sources each with the same average $\beta$ (i.e. ignoring any morphological variation throughout the galaxy and treating each star as though it has the same typical $\beta$ ) and with luminosity density function $\rho\left(R, z^{\prime}\right)$ is calculated to take the form

$$
V_{\beta}(r, z)=-2 \pi \beta \int_{0}^{\infty} d k \int_{0}^{\infty} d R \int_{-\infty}^{\infty} d z^{\prime} R \rho\left(R, z^{\prime}\right) J_{0}(k r) J_{0}(k R) e^{-k\left|z-z^{\prime}\right|}
$$

where $R, z^{\prime}$ are cylindrical source coordinates and $r$ and $z$ are the only observation point coordinates of relevance. Analogously, the net contribution of a disk of stars each with a linear $\gamma r / 2$ potential (i.e. ignoring any possible morphological variation in $\gamma$ ) is found to take the simple form

$$
\begin{gathered}
V_{\gamma}(r, z)=\pi \gamma \int d k d R d z^{\prime} R \rho\left(R, z^{\prime}\right) \\
\times\left[\left(r^{2}+R^{2}+\left(z-z^{\prime}\right)^{2}\right) J_{0}(k r) J_{0}(k R)-2 r R J_{1}(k r) J_{1}(k R)\right] e^{-k\left|z-z^{\prime}\right|}
\end{gathered}
$$

For a thin exponential disk with $\rho\left(R, z^{\prime}\right)=\Sigma(R) \delta\left(z^{\prime}\right)=\Sigma_{0} \exp (-\alpha R) \delta\left(z^{\prime}\right)$ where $R_{0}=$ $1 / \alpha$ is the disk scale length and $N=2 \pi \Sigma_{0} R_{0}^{2}$ is the total number of stars in the disk, it is possible to perform the integrations completely to yield

$$
V_{\beta}(r)=-\pi \beta \Sigma_{0} r\left[I_{0}(\alpha r / 2) K_{1}(\alpha r / 2)-I_{1}(\alpha r / 2) K_{0}(\alpha r / 2)\right]
$$


for the Newtonian contribution (Freeman (1970)), and

$$
\begin{aligned}
V_{\gamma}(r) & =\pi \gamma \Sigma_{0}\left\{\left(r / \alpha^{2}\right)\left[I_{0}(\alpha r / 2) K_{1}(\alpha r / 2)-I_{1}(\alpha r / 2) K_{0}(\alpha r / 2)\right]\right. \\
& \left.+\left(r^{2} / 2 \alpha\right)\left[I_{0}(\alpha r / 2) K_{0}(\alpha r / 2)+I_{1}(\alpha r / 2) K_{1}(\alpha r / 2)\right]\right\}
\end{aligned}
$$

for the net linear contribution. Differentiation then yields the remarkably compact expression

$$
\begin{gathered}
r V^{\prime}(r)=\left(N \beta \alpha^{3} r^{2} / 2\right)\left[I_{0}(\alpha r / 2) K_{0}(\alpha r / 2)-I_{1}(\alpha r / 2) K_{1}(\alpha r / 2)\right] \\
+\left(N \gamma \alpha r^{2} / 2\right) I_{1}(\alpha r / 2) K_{1}(\alpha r / 2)
\end{gathered}
$$

an expression which behaves asymptotically as $N \beta / r+N \gamma r / 2-3 N \gamma R_{0}^{2} / 4 r$ as would be expected. The coefficient $N \beta$ is usually identified as $M G / c^{2}$ with $M$ being taken to be the mass of the disk; and we see that for thin disks all departures from the standard Freeman result are embodied in the $\gamma$-dependent term in the simple and compact manner indicated.

\section{(3) Exponential Disks and Flat Rotation Curves}

In a recent comprehensive analysis of the $H I$ rotation curves of spiral galaxies (the more prominent HII optical data studies of the type pioneered by Rubin et. al. (1978) do not go out to a large enough number of scale lengths to show any substantive deviation from a standard luminous Newtonian behaviour (Kalnajs (1983), Kent (1986)) making the HI data the main probe of the outer reaches of the rotation curve), Casertano and van Gorkom (1991) have found that the data fall into essentially four general groups characterized by specific correlations between the maximum rotation velocity and luminosity; with the four groups being intermediate, compact bright, large bright, and dwarf galaxies. Thus as a first attempt at data fitting we have chosen to study one representative galaxy from each group, specifically the galaxies NGC3198, NGC2903, NGC5907, and DDO154. This will immediately enable us to test the flexibility of our theory, as well as confront the systematics apparent in dark matter fits to the same four groups where it is typically found that the more luminous the galaxy the proportionately less dark matter seems to be needed. 
The intermediate galaxy NGC3198 is particularly well-suited for testing theories since for it the data go out to the largest known number of surface brightness scale lengths; and, with the data being so flat, this galaxy is generally regarded as being prototypical. To model the galaxy we have followed van Albada et. al. (1985), and represented the surface brightness by a single exponential with a $1^{\prime}(=2.72 \mathrm{kpc})$ scale length. (This choice approximates Wevers et. al. (1986) $U^{\prime}, J$, and $F$ filter data with eyeball slopes of $R_{0}=$ $63^{\prime \prime}, 58^{\prime \prime}, 54^{\prime \prime}$ respectively at a $5 \%$ uncertainty level (the $F$ filter data have also been confirmed by Kent (1987)), while ignoring a spike in the very small angle region data, and also a possible truncation at the edge of the visible region). Moreover, the model ignores any modifications to the luminosity profile due to extinction or galactic dust infrared reprocessing. Following Begeman $(1987,1989)$ we have assigned a $z$-thickness to the disk according to the general analysis of van der Kruit and Searle (1981), so that the luminosity density function $\rho\left(R, z^{\prime}\right)$ needed for Eqs. (2) and (3) takes the separable form $\Sigma(R) \operatorname{sech}^{2}\left(z^{\prime} / z_{0}\right) / 2 z_{0}$ with $z_{0}=R_{0} / 5$. (The $z$-thickness structure of the disk is only significant at small radii where it serves to ensure that the inner part of the rotation curve is well fitted by the Newtonian contribution, thus making it possible to explore fully the effect of the linear potential on the outer region). Recognizing a $15 \%$ or so contribution to the visible mass density from the $H I$ gas itself, we have also included the gas as a matter source, and have found that, for model purposes, Wevers et. al. (1986) $H I$ surface density data can be well represented by a sum of three exponentials, viz. $\sigma_{H I}(R)=(37.0 \exp (-R / 2.23)+34.6 \exp (-R / 0.87)-68.2 \exp (-R / 1.21)) M_{\odot} / \mathrm{pc}^{2}(R$ is in arc minutes) with a total $H I$ mass (to infinity) of $5.2 \times 10^{9} M_{\odot}$, of which $4.9 \times 10^{9} M_{\odot}$ is observed in the explored $12^{\prime}$ region. Finally, again following Begeman, and also van Albada and Sancisi (1986), we have multiplied the $H I$ gas profile by a factor of 1.4 to allow for the presence of helium. With the model thus defined with only the two free parameters $N$ and $\gamma$ of the stars, we have generated the fit of Fig. (1) to Begeman's (1989) rotation curve data. (The fitting proved not to be sensitive to any thickness for the 
gas so we did not use one, while the fit was also insensitive to any deviations of the value of the gaseous $\gamma / \beta$ ratio from the stellar one). With $N$ essentially being constrained by the overall normalization of the stellar contribution, our best fit is found to yield a value of $3.8 \times 10^{10} M_{\odot}$ for the mass of the stars which is quite reasonable for a galaxy with quoted luminosity $L_{B}=9.0 \times 10^{9} L_{B \odot}$ (i.e. galactic mass to light ratio $M / L=4.2 M_{\odot} / L_{B \odot}$ ), with the obtained galactic mass in fact being a typical so called maximum disk mass in which the Newtonian term gets to be as large as it possibly can be. Additionally, we find that the coefficient of the net galactic linear term is given by $1 / \gamma_{\text {galaxy }}=1 / N \gamma_{\text {star }}=2.9 \times 10^{29}$ $\mathrm{cm}$ to yield a galactic gamma to light ratio $\gamma_{\text {galaxy }} / L_{B}=3.9 \times 10^{-40} / \mathrm{cm} / L_{B \odot}$. As we can see from Fig. (1), the contribution of the linear potential piece is remarkably reminiscent in shape to that of a typical dark matter contribution to galactic data fitting (see e.g. Kent (1987) for an extensive study); and intriguingly we find that the linear potential is competitive with the Newtonian one in a galaxy when $1 / \gamma_{\text {galaxy }}$ is of order the Hubble radius, the naive anticipation of Mannheim and Kazanas (1989) in their original study. Given this value for $\gamma_{\text {galaxy }}$, the inferred value for $\gamma_{\text {star }}$ is then $0.9 \times 10^{-40} \mathrm{~cm}^{-1}$, making the linear potential indeed negligible on solar system distance scales as initially required, with the linear potential only first becoming competitive with the Newtonian one galactically. (In passing we note that with such a small value for $\gamma$ the $\beta \gamma$ product terms in Eq. (1) are then rendered completely insignificant, a fact we had indicated earlier). As regards an assessment of the quality of our fitting, we should note that there are some still not fully understood discrepancies in the outer region (of order up to 7 km/s) between Begeman's data and Bosma's earlier 1978, 1981 data; while additionally Begeman quotes a maximum difference of $3 \mathrm{~km} / \mathrm{s}$ between his inner rotation curve and that of Hunter et al (1986). Moreover, we should also note that Begeman's last 2 data points (the farthest) actually use adopted values which are extrapolated from closer in ones. Also, there is even some indication in the data of a warp at the largest observed distances which we have not attempted to model. Finally, in general as regards rotation 
curve data, we note that velocities are measured at corresponding distances on the two sides (receding and approaching) of the galaxy, with differences between the obtained values usually constituting the (sole) quoted errors, even though such differences could well indicate that the galaxy actually has a symmetry lower than that of a disk (in which case the very extraction of the original mass density itself from the surface brightness data becomes slightly suspect). Thus fitting at the $5 \%$ (or even 10\%) level would seem to be acceptable, making our fitting quite adequate.

For the compact, bright galaxy NGC2903 Wevers et al (1986) find a stellar disk scale length of $2.0 \mathrm{kpc}\left(=1.08^{\prime}\right)$. For this galaxy the contribution of the gas is quite small $\left(M_{H I}=2 \times 10^{9} M_{\odot}\right)$, and introducing a stellar or a gaseous thickness was found to have no appreciable effect. Consequently we are able to fit the galaxy directly with Eq. (6) (using the same $\gamma / \beta$ ratio for stars and gas) to find the fit of Fig. (1) to Begeman's (1987) rotation curve data data. We find a fitted mass of $5.3 \times 10^{10} M_{\odot}$ for the stars to be compared with quoted luminosity $L_{B}=1.5 \times 10^{10} L_{B \odot}\left(M / L=3.5 M_{\odot} / L_{B \odot}\right)$, and a net galactic linear term given by $1 / \gamma_{\text {galaxy }}=1 / N \gamma_{\text {star }}=1.3 \times 10^{29} \mathrm{~cm}$, making $\gamma_{\text {star }}=1.4 \times 10^{-40} \mathrm{~cm}^{-1}$ and $\gamma_{\text {galaxy }} / L_{B}=5.1 \times 10^{-40} / \mathrm{cm} / L_{B \odot}$. As regards the fitting we note that this galaxy also has a warp at large distances which we have not attempted to model. Also, in his original dark matter fit Begeman (1987) found a lot of scatter in the inner rotation curve (he actually settled for an eye-ball fit rather than a least squares one), prompting him to suggest that there might be an additional stellar component in the not well explored close in region. If there is, then in the present theory such a component would also contribute in the outer region because of the linear term.

Until very recently the large, bright galaxy NGC5907 had actually had a severe fitting problem because the original surface brightness data of van der Kruit and Searle (1981) were simply completely incompatible with the rotation curve data (van Albada and Sancisi (1986)) in the inner region where the stellar Newtonian potential should dominate. This situation has only recently been rectified by Barnaby and Thronson (1992a) who find (using 
a different filter) a completely different surface brightness curve, one which does nicely fit the inner region (Barnaby and Thronson (1992b)). Barnaby and Thronson find that the stellar disk is well parametrized by $\rho\left(R, z^{\prime}\right)=\Sigma_{0} \exp \left(-R / R_{0}\right) \operatorname{sech}\left(z^{\prime} / z_{0}\right) / \pi z_{0}$ where $R_{0}$ $=4.0 \mathrm{kpc}\left(=1.22^{\prime}\right)$, and $R_{0} / z_{0}=9.2$. Additionally they find a close in (and thus easy to miss) central region stellar bulge with a luminosity $5 \%$ of that of the disk which they parametrize by a modified Hubble profile $\left(\sim 1 /\left(R^{2}+R_{0}^{2}\right)\right)$ with scale length $R_{0}=0.07^{\prime}$. The total stellar contribution to the rotation curve is exhibited in Fig. (1). We find the mass of the disk to be $1.1 \times 10^{11} M_{\odot}$ and that of the bulge to be $1.7 \times 10^{10} M_{\odot}$ with the quoted luminosity of the galaxy being $L_{B}=1.8 \times 10^{10} L_{B \odot}\left(M / L=6.1 M_{\odot} / L_{B \odot}\right.$ for the disk). For the linear term we find (we use the same $\gamma / \beta$ ratio for the disk and bulge) $1 / \gamma_{\text {galaxy }}=1 / N \gamma_{\text {star }}=1.7 \times 10^{29} \mathrm{~cm}$, making $\gamma_{\text {star }}=5.5 \times 10^{-41} \mathrm{~cm}^{-1}$ and $\gamma_{\text {galaxy }} / L_{B}=3.2 \times 10^{-40} / \mathrm{cm} / L_{B \odot}$, to give values which are comparable with those of the other galaxies.

For the dwarf irregular DDO154 Carignan and Freeman (1988) and Carignan and Beaulieu (1989) have determined both the rotation curve and the surface brightness data. The stellar component is fit by a disk with scale length $0.43^{\prime}$ (corresponding to $R_{0}=0.5$ kpc if the galaxy is at a distance $D=4 \mathrm{Mpc}$ - it may be at $D=10 \mathrm{Mpc}$, see below); while the gas is well fitted by $\sigma_{H I}(R)=(31.6 \exp (-R / 1.42)-25.7 \exp (-R / 1.08)) M_{\odot} / \mathrm{pc}^{2}(R$ is in arc minutes) with a total $H I$ mass (to infinity) of $2.8 \times 10^{8} M_{\odot}$ (at $D=4 \mathrm{Mpc}$ ), $93 \%$ of which is observed in the explored region. Since the gas turns out to be the main gravitational component we see that the observed region corresponds to about 4.5 leading $1.4^{\prime}$ gas scale lengths. In the central galactic region the stellar surface brightness is not yet fully explored (it actually appears to be flattening off, meaning that an exponential disk could be overestimating the inner surface brightness). Consequently in order to fit the inner rotation curve we have found it necessary to give the stellar component a $\operatorname{sech}^{2}\left(z^{\prime} / z_{0}\right) / 2 z_{0}$ thickness with $z_{0}=R_{0}$ (stellar)/3. Additionally, we have allowed the stellar and gaseous $\gamma / \beta$ ratios to vary independently (there is no immediate reason why they should be the 
same, anymore than the mass to light ratios of bulges and disks of a given galaxy should be the same). We have not included the contribution of gas pressure (a $5 \%$ or so effect which is completely ignorable in galaxies where stars dominate the dynamics), nor have we considered any random motions of the gas (a 1 or $2 \%$ effect), or an apparent galactic warp. Also we note that the very last rotation curve data point was only determined on one side (the receding one) of the galaxy and its inferred velocity is not independent of those of the two immediately previous points. Our best fit at $D=4 \mathrm{Mpc}$ is presented in Fig. (1) with stellar mass $6.8 \times 10^{7} M_{\odot}$ for a quoted luminosity at that distance of $L_{B}=5.0 \times 10^{7} L_{B \odot}$ $\left(M / L=1.4 M_{\odot} / L_{B \odot}\right)$. For the stars we find $1 / \gamma_{\text {galaxy }}=1 / N \gamma_{\text {star }}=4.0 \times 10^{29} \mathrm{~cm}$, making $\gamma_{\text {star }}=3.7 \times 10^{-38} \mathrm{~cm}^{-1}$ and $\gamma_{\text {galaxy }} / L_{B}=5 \times 10^{-38} / \mathrm{cm} / L_{B \odot}$, while the best value for $\gamma_{g a s}$ is found to be zero (i.e. much smaller than the stellar contribution). Now while Carignan and coworkers favor putting DDO154 at $D=4 \mathrm{Mpc}$, we note that they also indicated a possible adopted distance at $D=10 \mathrm{Mpc}$ which Krumm and Burstein (1984) favor, this being the distance at which the Tully-Fisher relation is obeyed. Since the amount of gas is 6.25 times bigger at the larger distance, it would then be totally dominant. (While we shall continue to use the same $\sigma_{H I}(R)$ as before after scaling up to the larger distance, we note that Krumm and Burstein actually obtained a leading scale length of $2.5^{\prime}$ ). At $D=10 \mathrm{Mpc}$ the fit is found to be insensitive to any stellar or gaseous disk thickness, with the thin disk approximation then yielding the fit of Fig. (1). We find the stellar mass to be $7.4 \times 10^{7} M_{\odot}$. Additionally we find that for the stars $1 / \gamma_{\text {galaxy }}=1 / N \gamma_{\text {star }}=1.4 \times 10^{30}$ $\mathrm{cm}$, making $\gamma_{\text {star }}=9.9 \times 10^{-39} \mathrm{~cm}^{-1}$, while the best value for $\gamma_{g a s}$ is again found to be zero. (Actually, the fits at both the candidate distances can even be improved in the outer region if we allow $\gamma_{g a s}$ to go negative. This would be somewhat difficult to understand, though without a dynamical theory for $\gamma$ it cannot yet be excluded). Since our theory, the standard flat dark matter theory, and MOND would all eventually overshoot the data if the suggested large distance decline in the DDO154 rotation curve were to be confirmed, further observational study of this point might prove interesting. 
In our fitting we see a reflection of the general luminosity trend found in dark matter fits, with both the relative Newtonian contribution and the inferred galactic mass to light ratios increasing with luminosity. Additionally, and intriguingly we find that the values obtained for $1 / \gamma_{\text {galaxy }}$ are remarkably close to each other and to the Hubble radius suggesting some possible common underlying dynamics. (Perhaps $\gamma_{\text {galaxy }}$ sets or is set by the scale at which galaxies can fluctuate out of an initial cosmological background). For the three regular galaxies NGC3198, NGC2903, and NGC5907 we find that the mass to light ratios are comparable to each other and likewise their gamma to light ratios, to thus suggest only a mild morphological dependence to the average stellar $\beta$ and $\gamma$ parameters used as input for Eqs. (2) and (3). (While the mass to light ratio is assumed to be uniform within a given galaxy, the actual numerical value of this ratio is not apparently universal for all galaxies suggesting that galaxies do not all have the same universal mix of stars and/or the same typical average stellar $\beta$. A similar situation should thus be expected to obtain for the galactic gamma to light ratio). We note that the irregular galaxy DDO154 does not show the same galactic gamma to light ratio as the other three galaxies. It is not clear whether this is a fundamental issue or whether perhaps the galaxy has an anomalous mix of stars. As regards this issue we recall that both the dark matter and MOND fits of Begeman et al (1991) to the same galaxy find atypically small mass to light ratios. Thus dwarf irregulars may be fundamentally different, though of course trying to extract out stellar parameters in a galaxy where the stars do not dominate may not be completely reliable. With regard to our fitting, we see that while our fitting is yielding flat rotation curves, it is doing so in a theory in which rotation curves must eventually rise. This stands in marked contrast to the asymptotically flat behaviour expected both in MOND and in the isothermal gas sphere model of dark matter with its asymptotically logarithmic galactic potential. (Other, less popular, asymptotic alternatives for dark matter are considered in van Albada et al (1985)). Since one may unfortunately run out of galaxy before possibly seeing any such rise, perhaps the sharpest difference between linearly rising and logarithmic potentials 
may emerge at the slightly larger distance scale associated with gravitational lensing where such differences might even be pronounced, thus making a study of the (so far unknown) conformal gravity lensing predictions in the non-asymptotically flat geometry of Eq. (1) quite urgent.

We believe that we have thus established the candidacy (at least) of fourth order gravity by working up from the non-relativistic limit; and since the conformal theory has already been shown to possess no flatness problem (Mannheim (1992)) and thus not require any cosmological dark matter, and since the linear potential has also been shown to be capable of yielding galactic stability without the need for dark matter (Christodoulou (1991)), we see that both cosmologically and galactically it might turn out to be the case that luminous matter is the major constituent of the Universe after all.

The author would like to thank D. Kazanas, D. Christodoulou and J. Taylor for stimulating discussions, and D. Barnaby, K. Begeman, C. Carignan, S. Casertano, S. Kent and R. Sancisi for some very helpful communications. This work has been supported in part by the Department of Energy under grant No. DE-FG02-92ER40716.00. 


\section{References}

Barnaby, D., and Thronson, H. A. 1992a, A. J. 103, 41.

Barnaby, D., and Thronson, H. A. 1992b, B.A.A.S. 24, 809.

Begeman, K. G. 1987, Ph. D. Thesis, Gronigen University.

Begeman, K. G. 1989, A. A., 223, 47.

Begeman, K. G., Broeils, A. H., and Sanders, R. H. (1991), Mon. Not. R. Astron. Soc. 249,523 .

Bosma, A. 1978, Ph. D. Thesis, Gronigen University.

Bosma, A. 1981, A. J., 86, 1791.

Carignan, C., and Freeman, K. C. 1988, Ap. J. (Letters) 332, 33.

Carignan, C., and Beaulieu, S. 1989, Ap. J. 347, 760.

Casertano, S. 1983, Mon. Not. R. Astron. Soc. 203, 735.

Casertano, S., and van Gorkom, J. H. 1991, A. J., 101, 1231.

Christodoulou, D. M. 1991, Ap. J., 372, 471.

Freeman, K. C. 1970, Ap. J. 160, 811.

Hunter, D. A., Rubin, V. C., and Gallagher III, J. S. 1986, A. J. 91, 1086.

Kalnajs, A. J. 1983, in Internal Kinematics and Dynamics of Disk Galaxies, IAU Symposium No. 100, ed. E. Athanassoula (Reidel, Dordrecht), p. 87.

Kazanas, D., and Mannheim, P. D. 1991, Ap. J. Suppl. Ser., 76, 431.

Kent, S. M. 1986, A. J. 91, 1301.

Kent, S. M. 1987, A. J. 93, 816.

Krumm, N., and Burstein, D. 1984, A. J. 89, 1319.

Mannheim, P. D. 1990, Gen. Rel. Grav., 22, 289.

Mannheim, P. D. 1992, Ap. J., 391, 429.

Mannheim, P. D. 1993, Gen. Rel. Grav. (in press).

Mannheim, P. D., and Kazanas, D. 1989, Ap. J., 342, 635.

Mannheim, P. D., and Kazanas, D. 1991, Phys. Rev., D44, 417. 
Mannheim, P. D., and Kazanas, D. 1992, Newtonian limit of conformal gravity and the lack of necessity of the second order Poisson equation, UCONN-92-4, unpublished.

Milgrom, M. 1983, Ap. J. 270, 365; 371, 384.

Rubin, V. C., Ford W. K., and Thonnard, N. 1978, Ap. J. (Letters), 225, L107.

Sanders, R. H. 1990, A. A. Rev., 2, 1.

Toomre, A. 1963, Ap. J., 138, 385.

van Albada, T. S., Bahcall, J. N., Begeman, K. G., and Sancisi, R., 1985, Ap. J., 295, 305.

van Albada, T. S., and Sancisi, R., 1986, Phil. Trans. R. Soc., A320, 447.

van der Kruit, P. C., and Searle L., 1981, A. A., 95, 105.

Wevers, B. M. H. R., van der Kruit, P. C., and Allen, R. J. 1986, A. A. Suppl. Ser., 66, 505.

\section{Figure Caption}

Figure (1). The calculated rotational velocity curves associated with the metric of Eq. (1) for the four representative galaxies, the intermediate sized NGC3198, the compact bright NGC2903, the large bright NGC5907, and the dwarf irregular DDO154 (at two possible adopted distances). In each graph the bars show the data points with their quoted errors, the full curve shows the overall theoretical velocity prediction (in $\mathrm{km} / \mathrm{s}$ ) as a function of distance (in arc minutes) from the center of each galaxy, while the two indicated dotted curves show the rotation curves that the separate Newtonian and linear potentials of Eq. (1) would produce when integrated over the luminous matter distribution of each galaxy. No dark matter is assumed. 